\title{
Mitteilungen der GARPS
}

Gastroenterologe $2021 \cdot 16: 208-209$

https://doi.org/10.1007/s11377-021-00526-2

(c) Springer Medizin Verlag GmbH, ein Teil von Springer Nature 2021

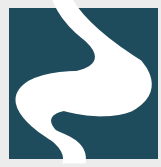

GARPS

Gastroenterologische Arbeitsgemeinschaft

Rheinland-Pfalz /Saarland
Gastroenterologische

Arbeitsgemeinschaft

Rheinland-Pfalz/Saarland (GARPS)

Kastanienweg 4

67146 Deidesheim

Tel. 06326/962887

www.garps.de

Redaktion V.i.S.d.P.

Prof. Dr. P. Galle

I. Medizinische Klinik und Poliklinik

Langenbeckstraße 1

55131 Mainz
Prof Dr. R. Jakobs

Medizinische Klinik C

Klinikum der Stadt Ludwigshafen

Bremserstraße 79

67063 Ludwigshafen

Prof. Dr. F. Lammert

Klinik für Innere Medizin II

Universitätsklinikum des Saarlandes

Kirrberger Straße 100

66421 Homburg/Saar

T. Adams ${ }^{1} \cdot$ J. Lehmann ${ }^{1} \cdot$ M. Krawczyk ${ }^{1,2} \cdot$ F. Lammert ${ }^{1,3}$.

M.C. Reichert ${ }^{1}$

${ }^{1}$ Klinik für Innere Medizin II (Gastroenterologie und Endokrinologie), Universitätsklinikum des Saarlandes, Homburg

${ }^{2}$ Laboratory of Metabolic Liver Diseases, Centre for Preclinical Research, Department of General, Transplant and Liver Surgery,

Medical University of Warsaw, Warsaw, Poland

${ }^{3}$ Medizinische Hochschule Hannover (MHH), Hannover

\section{Gastroenterologie und SARS-CoV2: Ein Jahr auf der COVID-19 Normalstation}

Vor genau einem Jahr veröffentlichten wir in dieser Zeitschrift (Ausgabe 3/2020) in den Mitteilungen der GARPS unseren Kommentar zur COVID-19-Pandemie [1]. Zum damaligen Zeitpunkt standen wir am Beginn der Pandemie, die inzwischen weltweit mehr als 120 Millionen Menschen betroffen und zu mindestens 2,7 Millionen Toten geführt hat [2]. Während des vergangenen Jahres wurde eine Station unserer Klinik zur Behandlung und Pflege von mit SARS-CoV2 infizierten Patienten aus allen anderen Kliniken des Universitätsklinikums genutzt. Über dieses Jahr hinweg haben wir über 170 Patienten mit COVID-19 auf dieser Normalstation behandelt. Neben einem Infektionscluster mit Bezug zu einer Pflegeeinrichtung haben wir zahlreiche Patienten mit (zumeist erworbener) Immunschwäche behandelt. Hierzu zählen insbesondere transplantierte Patien- ten und Patienten mit hämatologischen Vorerkrankungen und -therapien. In anderen Fällen stand nicht COVID-19 im Vordergrund, denn gerade aus den operativen Fächern wurden einzelne Patienten, die aus krankenhaushygienischen Gründen auf unsere Station verlegt wurden, betreut. Im vorliegenden Beitrag möchten wir unsere Erfahrungen aus den vergangenen 12 Monaten vorstellen.

Bei den Patienten traten die beiden bekannten Phasen der COVID-19 Erkrankung auf: die häufige und durch eine hohe Virusreplikation gekennzeichnete Frühphase der Erkrankung und die immunologisch vermittelte Spätphase der Erkrankung. Beide Phasen betreffen unterschiedliche Organsysteme in variabler Weise.

Zunächst beginnt die virämische Phase, diese ähnelt anderen viralen Erkrankungen wie der saisonalen Influenza und zeigt wie diese ein heterogenes Bild von milden oder oligosymptomatischen Verläufen bis hin zu schweren Verläufen mit hohem Fieber. Neben den typischen grippalen Symptomen wie Fieber, Myalgien und respiratorischen Symptomen waren auch hier sehr häufig neurologische Symptome vorzufinden, die von den charakteristischen Geruchsund Geschmacksstörungen bei eher jungen Menschen bis hin zu hypoaktiv-deliranten Zuständen bei älteren Erkrankten reichen. In allen Altersgruppen ist häufig eine ausgeprägte Fatigue-Symptomatik zu beobachten. Gastrointestinale Beschwerden in Form von Übelkeit, Erbrechen, Inappetenz und abdominellen Schmerzen in verschiedener Ausprägung sind häufig vorhanden. Es zeigt sich laborchemisch zumeist ein hepatitisches, aber auch ein cholestatisches Schädigungsmuster, der Verlauf war in der Regel selbstlimitiert, und die Leberwerte fielen meist spontan. Da der von SARS-CoV2 genutzte ACE2Rezeptor fast ubiquitär und auch von Cholangio- und Hepatozyten exprimiert wird, könnte hier zumindest partiell eine direkte Schädigung dieser Zellen durch das Virus vorliegen [3].

Neben der Symptomatik des oberen Gastrointestinaltrakts traten häufig Diarrhoen als Symptome des unteren Gastrointestinaltrakts auf. Hierzu passend konnten wir auch bei unseren Patienten wiederholt erhöhte Calprotectinkonzentrationen im Stuhl nachweisen [4].
Wie in der Literatur dokumentiert, konnten wir bei einem Teil unserer Patienten die Dauer und Symptomlast der virämischen Phase durch die verfügbaren $\mathrm{Me}$ dikamente wie Remdesivir, Rekonvaleszentenplasma und Antikörper positiv beeinflussen.

Der virämischen Phase folgt die immunologische Phase, die durch die Folgen der Endothelitis mit erhöhten Zytokinen, vor allem Interleukin-6, gekennzeichnet ist [5]. Im klinischen Alltag haben sich bei uns ein Abfall der Lymphozyten sowie ein Anstieg des CRP, der D-Dimere und des IL-6 als Hinweise für einen schlechten Verlauf bewährt. Als häufigste Komplikation ist das ARDS zu nennen, dass trotz Ausschöpfung aller intensivmedizinischen Maßnahmen mit einer hohen Mortalität von mehr als $50 \%$ einhergeht. Die immunvermittelten Phänomene zeigen sich in Form thromboembolischen Ereignissen. Wir beobachten neben Lungenembolien gehäuft venöse Thrombosen und auch arterielle Verschlüsse. Daher muss bei COVID-19 unbedingt schon bei Aufnahme individuell eine prophylaktische Antikoagulation etabliert werden, bei weiteren Risikofaktoren gegebenenfalls eine therapeutische Antikoagulation. Zudem beobachteten wir bei einzelnen Patienten ein rasch progredientes Nierenversagen, das eine intermittierende Dialyse erforderte. Diese wurde auf der Station durchgeführt. In diesen Fällen war aufgrund der Vorerkrankungen eine 
Vorschädigung der Niere möglich, auch hier konnte jedoch in der interdisziplinären Abklärung regelhaft kein Hinweis auf eine prä- oder postrenale oder sonstige Ursachen gefunden werden. Auch wenn keine histologische Sicherung erfolgen konnte, erscheint - nicht zuletzt aufgrund des zeitlichen Zusammenhangs - eine COVID-19-bedingte Nierenschädigung möglich. Histopathologisch konnten hierzu in Voruntersuchungen postmortal bei an COVID-19 Erkrankten spezifische Veränderungen nachgewiesen werden [5].

Das Auftreten der einen schwereren Verlauf kennzeichnenden Immunologischen Phase tritt gehäuft bei Risikofaktoren auf. Vor allem das höhere Lebensalter und Komorbiditäten spielen hier eine Rolle, aber genetische Faktoren werden ebenfalls diskutiert. Unsere Erfahrung zeigt, dass hier der rechtzeitige Einsatz von Dexamethason nach den aktuellen Empfehlungen des Robert-Koch-Instituts [6] bei vielen Patienten ein gutes klinisches Ansprechen zeigt.

Auf unserer Station waren $\mathrm{Pa}$ tienten mit erworbener Immunschwäche weniger häufig vor einem schweren Verlauf betroffen, und wir beobachten über mehrere Wochen eine persistierende Virämie. Bei den Erkrankten, die im Vorfeld eine solide Organtransplantation erhalten hatten, konnte bei allen Patienten unter Reduktion der immunsuppressiven Therapie eine anhaltende Viruselemination erreicht werden. Von den Erkrankten nach einer soliden Organtransplantation (in absteigender Häufigkeit Nieren-, Lungen-, Herz- und Lebertransplantationen), die bei uns stationär aufgenommen wurden, mussten wir nur einen Organverlust (Niere) hinnehmen. In diesem Fall lag jedoch bereits eine chronische Abstoßungsreaktion im Vorfeld vor.

Eine andere Gruppe mit protrahiertem Verlauf waren Patienten mit einem Antikörpermangel bei B-Zell-Depletion. In unserem Zentrum lag den erworbenen
Mangelzuständen die Therapie mit einem gegen B-Lymphozyten gerichteten monoklonalen Antikörper (Rituximab oder Ocrelizumab) zugrunde. Der überwiegende Teil zeigte rezidivierende Fieberschübe ohne weitere ausgeprägte Symptome. Interessanterweise zeigten sich bei diesen Patienten eine nur geringe Viruslast mit teilweise sogar negativer SARS-CoV2-PCR in einzelnen Nase-Rachen-Abstrichen oder isoliertem Nachweis in der bronchoalveolären Lavage. Die Patienten wurden mit Rekonvaleszentenplasma und/oder Remdesivir (bis zu 10 Tage) behandelt. Bei allen Patienten konnte letztlich ein dauerhaftes virologisches und klinisches Ansprechen erreicht werden, welches bei einem Patienten in der Viruskultur bestätigt wurde. Zum damaligen Zeitpunkt bestand noch kein Zugang zu gegen SARS-CoV2 gerichteten monoklonalen Antikörpern, diese stellen aber jetzt - insbesondere, wenn sehr frühzeitig nach der Infektion eingesetzt - eine erfolgversprechende Therapieoption dar.

\section{Literatur}

1. Lehmann J, Lepper PM, Krawczyk M (2020) COVID-19 Pandemie und Gastroenterologie: Update. Gastroenterologe 15:246-247

2. Dong E, Du H, Gradner L et al (2020) An interactive web-based dashboard to track COVID-19 in real time. Lancet Infect Dis 20:533-534

3. Nardo AD, Schneeweiss-Gleixner $M$, Bakail M et al (2020) Pathophysiological mechnisms of liver injury in COVID-19. Liver Int 41:20-32

4. Effenberger M, Grabherr F, Mayr L et al (2020) Faecal calprotectin indicates intestinal inflammation in COVID-19. Gut 69:1543-1544

5. Leisman DE, Ronner L, Pinotti R et al (2020) Cytokine elevation in severe and critical COVID-19: a rapid systematic review, meta-analysis, and comparison with other inflammatory syndromes. Lancet Respir Med 8:1233-1244

6. Feldt T, Guggemos W, Heim K et al (2021) Hinweise zu Erkennung, Diagnostik und Therapie von Patienten mit COVID-19. www.rki.de/covid19-therapie-stakob. Zugegriffen: 31. März 2021 